\title{
The 18S rRNA dimethylase Dim1p is required for pre-ribosomal RNA processing in yeast
}

\author{
Denis Lafontaine, ${ }^{1,2}$ Jean Vandenhaute, ${ }^{2}$ and David Tollervey ${ }^{1}$ \\ ${ }^{1}$ European Molecular Biology Laboratory (EMBL), D-69117 Heidelberg, Germany; ${ }^{2}$ Facultés Universitaires N-D de la Paix, \\ B-5000 Namur, Belgium
}

\begin{abstract}
The $m_{2}^{6} A_{1779} m_{2}^{6} A_{1780}$ dimethylation at the $3^{\prime}$ end of the small subunit rRNA has been conserved in evolution from bacteria to eukaryotes. The yeast $18 \mathrm{~S}$ rRNA dimethylase gene DIM1 was cloned previously by complementation in Escherichia coli and shown to be essential for viability in yeast. A conditional GAL10::dim1 strain was constructed to allow the depletion of Dim1p from the cell. During depletion, dimethylation of the pre-rRNA is progressively inhibited and pre-rRNA processing at cleavage sites A1 and A2 is concomitantly lost. In consequence, the mature $18 \mathrm{~S}$ rRA and its $20 \mathrm{~S}$ precursor drastically underaccumulate. This has the effect of preventing the synthesis of nonmethylated rRNA. To test whether the processing defect is a consequence of the absence of the dimethylated nucleotides or of the Dim1p dimethylase itself, a cis-acting mutation was created in which both dimethylated adenosines are replaced by guanosine residues. Methylation cannot occur on this mutant pre-rRNA, but no clear pre-rRNA processing defect is seen. Moreover, methylation of the wild-type pre-rRNA predominantly occurs after cleavage at sites $A 1$ and A2. This shows that formation of the $m_{2}^{6} A_{1779} m_{2}^{6} A_{1780}$ dimethylation is not required for pre-rRNA processing. We propose that the binding of Dimlp to the pre-ribosomal particle is monitored to ensure that only dimethylated pre-rRNA molecules are processed to $18 \mathrm{~S}$ rRNA.
\end{abstract}

Key Words: Methylation; RNA processing; ribosome synthesis; rRNA; yeast]

Received February 7, 1995; revised version accepted August 21, 1995.

Ribosomes have a complex biosynthetic pathway requiring the processing and modification of the ribosomal RNAs (rRNAs) and their assembly with ribosomal proteins (r-proteins) (for review, see Warner 1989; Raué and Planta 1991; Woolford and Warner 1991; Woolford 1991). In eukaryotic cells, most steps in ribosome synthesis occur in a specialized nuclear compartment, the nucleolus.

The four mature rRNA species are produced both by RNA polymerase I and polymerase III (Pol I and Pol III). Pol I transcribes a large precursor / the $35 \mathrm{~S}$ pre-rRNA in yeast) that undergoes a series of endo- and exonucleolytic processing reactions to yield three mature rRNA species, the $18 \mathrm{~S}$ rRNA component of the $40 \mathrm{~S}$ ribosomal subunit, and the $5.8 \mathrm{~S}$ and $25 \mathrm{~S}$ rRNA components of the $60 \mathrm{~S}$ ribosomal subunit. The structure of the yeast rDNA genes and a current model of the major yeast pre-rRNA processing pathway are presented in Figure 1 . The remaining rRNA species, the $5 \mathrm{~S}$ rRNA component of the 60S ribosomal subunit, is transcribed by Pol III.

Shortly after transcription, the pre-RNA is modified both by methylation and formation of pseudouridine, with all known pre-rRNA modifications occurring in the mature rRNA regions. In yeast, most of the 67 methyl groups are linked to the $2^{\prime}-\mathrm{O}$ of ribose moieties but 12 are attached to bases 16 in each of the $18 \mathrm{~S}$ and $25 \mathrm{~S}$
rRNAs). Base modification, unlike 2'-O-methylation, occurs mainly at later stages in ribosome biogenesis (Brand et al. 1977; Klootwijk and Planta 1989|. Almost all of the modified nucleotides that have been localized lie in the evolutionarily conserved regions of the rRNAs that are brought together in the functional center of the ribosome (for review, see Maden 1990; Brimacombe 1993).

Many of the methylated nucleotides present in eukaryotic rRNA are conserved from yeast to humans, arguing that they play some function that is both conserved and important. However, in no case is it known what role these modifications actually play in either the biogenesis or function of eukaryotic ribosomes. The requirement for methylation to allow the processing of eukaryotic pre-rRNA is also unclear. Methionine deprivation of HeLa cells leads to failure of the final stages of rRNA maturation (Vaughan et al. 1967). However, inhibition of methylation by treatment with ethionine, a competitive inhibitor of $S$-adenosylmethionine biosynthesis, allows partial (Swann et al. 1975; Wolf and Schlessinger 1977) or complete (Caboche and Bachellerie $1977)$ processing at the normal sites although with low efficiency. In yeast, the nop 1-3 mutant, which is inhibited in nucleolar pre-rRNA methylation, does not block pre-rRNA processing (Tollervey et al. 1993). Moreover, 
A

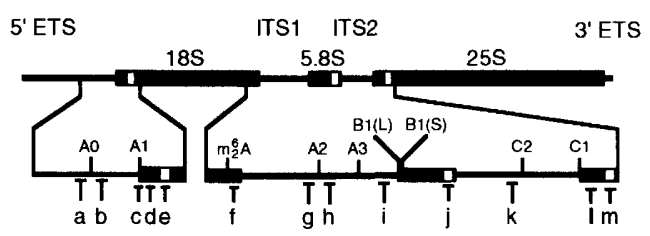

B

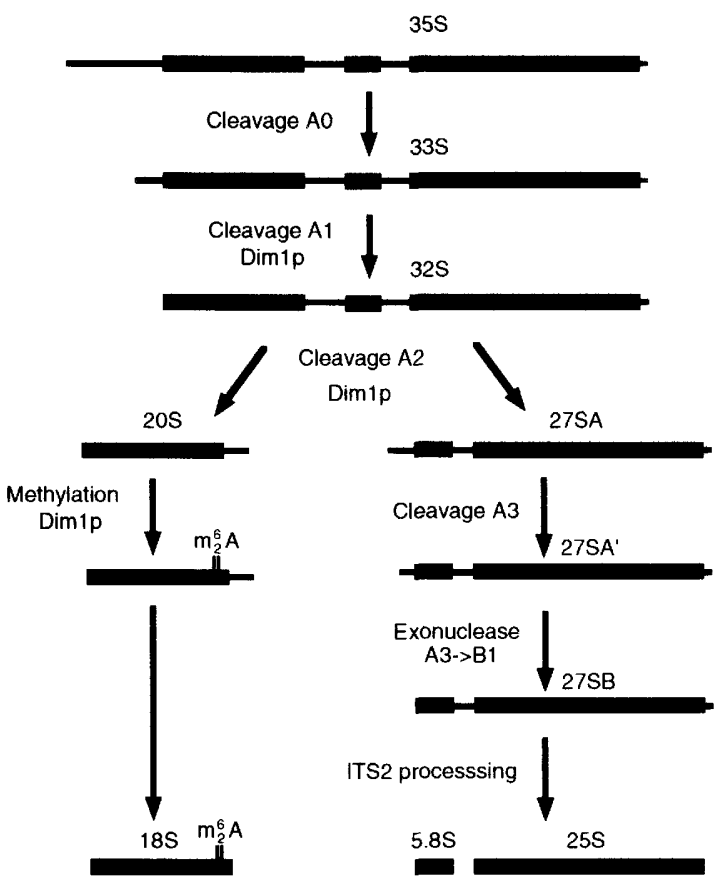

C
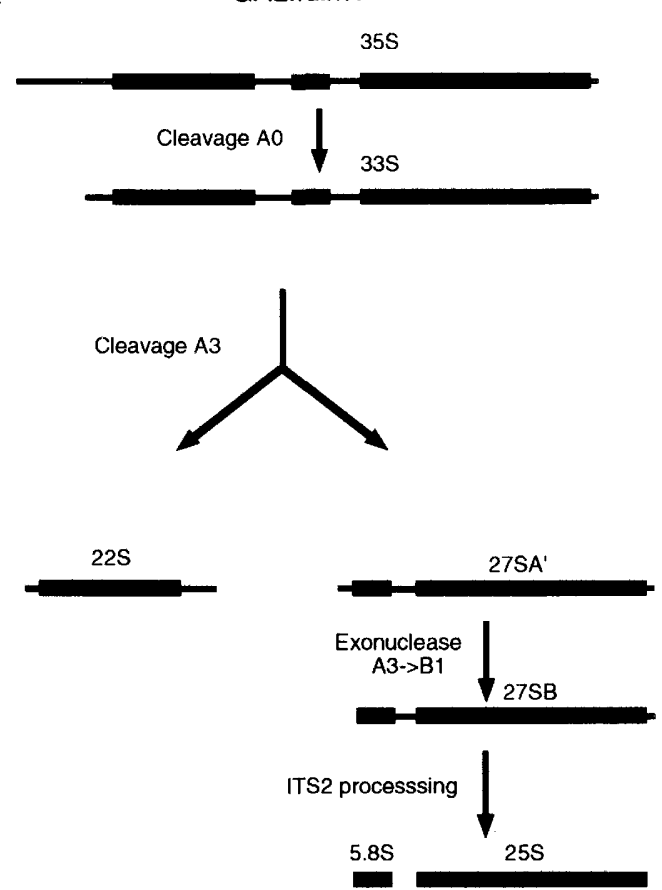

Figure 1. Structure of the yeast pre-rRNA and processing pathway. (A) Organization of the $35 \mathrm{~S}$ pre-rRNA. In the $35 \mathrm{~S}$ prerRNA, the $18 \mathrm{~S}, 5.8 \mathrm{~S}$, and $25 \mathrm{~S}$ rRNA genes are preceded and followed by noncoding sequences, the $5^{\prime}$ and $3^{\prime}$ external transcribed spacers $\left(5^{\prime}\right.$ ETS and $3^{\prime}$ ETS $)$, and separated by internal transcribed spacers 1 and 2 (ITS1 and ITS2). The pre-rRNA cleavages sites are indicated in uppercase, and oligonucleotides used for Northern hybridization and primer extension in lowercase. The dimethylation site is indicated as $m_{2}^{6} \mathrm{~A}$. $(B)$ The yeast pre-rRNA processing pathway. The $35 \mathrm{~S}$ pre-rRNA is successively cleaved at site $\mathrm{A} 0$ in the $5^{\prime} \mathrm{ETS}$, at site $\mathrm{Al}$, the $5^{\prime}$ end of mature 18S rRNA, and at site A2 in ITS1. The first two cleavages yield the $33 \mathrm{~S}$ and $32 \mathrm{~S}$ pre-rRNAs, respectively. Cleavage at site A2 splits the $32 \mathrm{~S}$ precursor into the $20 \mathrm{~S}$ and $27 \mathrm{SA}$ pre-rRNA that contain the RNA components of the small and large ribosomal subunits, respectively. The $20 \mathrm{~S}$ pre-rRNA is reported to be exported to the cytoplasm where $3^{\prime}$ processing generates the mature $18 \mathrm{~S}$ rRNA. The $27 \mathrm{SA}$ pre-rRNA is processed into $27 \mathrm{SA}^{\prime}$ pre-rRNA by cleavage at site $\mathrm{A} 3$ by RNase MRP. A3 acts as the entry site for an exonuclease activity, which generates the $5^{\prime}$ end of the $27 \mathrm{SB}(\mathrm{S})$ pre-rRNA at site $\mathrm{Bl}(\mathrm{S})$. This site is also the $5^{\prime}$ end of the mature $5.8 \mathrm{~S}(\mathrm{~S})$ rRNA, the major, short form of the $5.8 \mathrm{~S}$ rRNA. Subsequent cleavages in ITS2 generate the mature 5.8S(S) and 25S rRNAs. For simplicity, only the major processing pathway from $27 \mathrm{SA}$ to $5.8 \mathrm{~S}(\mathrm{~S})$ and $25 \mathrm{~S}$ rRNA is shown; an alternative pathway generates the minor $5.8 \mathrm{~S}$ (L) rRNA, which is 7-8 nucleotides $5^{\prime}$ extended (for a review of the pre-rRNA processing pathway, see Morrissey and Tollervey 1995). Reactions that require Dimlp are indicated. $(C)$ Pre-rRNA processing in the GAL:: $\operatorname{dim} 1$ strain. The $35 \mathrm{~S}$ prerRNA is cleaved normally at site A0. The $33 \mathrm{~S}$ pre-rRNA accumulates and is cleaved at site A3, yielding the $27 \mathrm{SA}^{\prime}$ pre-rRNA that is processed to $5.8 \mathrm{~S}(\mathrm{~S})$ and $25 \mathrm{~S}$ and an aberrant $22 \mathrm{~S}$ prerRNA that is not processed to $18 \mathrm{~S}$ rRNA.

in wild-type yeast cells treated with ethionine, processing of the $35 \mathrm{~S}$ pre-rRNA still occurs (Tollervey et al. 1993). The nop1-3 mutation does not, however, inhibit the late methylation of the $20 \mathrm{~S}$ pre-rRNA at $\mathrm{m}_{2}^{6} \mathrm{~A}_{1779} \mathrm{~m}_{2}^{6} \mathrm{~A}_{1780}$ (Tollervey et al. 1993; D. Tollervey, unpubl.). Finally, yeast pet56 mutants, which lack a ribose methylation in the mitochondrial large subunit rRNA, are defective in production of the large ribosomal subunit (Sirum-Connolly and Mason 1993).

Although the rRNAs of all organisms are methylated, the precise nucleotides that are modified are not generally conserved from eukaryotes to bacteria. An exception is the $\mathrm{m}_{2}^{6} \mathrm{~A}_{1518} \mathrm{~m}_{2}^{6} \mathrm{~A}_{1519} / \mathrm{m}_{2}^{6} \mathrm{~A}_{1779} \mathrm{~m}_{2}^{6} \mathrm{~A}_{1780}$ sequence (using, respectively, the Escherichia coli and the Saccharomyces cerevisiae numbering systems), localized in the loop of the hairpin helix 45 , at the very $3^{\prime}$ end of the small subunit rRNA. Not only the modification but the whole structure of this region is highly conserved (van Knippenberg et al. 1984). The functional role of the $\mathrm{m}_{2}^{6} \mathrm{Am}_{2}^{6} \mathrm{~A}$ modification has been studied extensively in $E$. coli, (for review, see van Knippenberg 1986) using mutants in $\operatorname{ksg} A$, which encodes the dimethylase (van Buul and van Knippenberg 1985); $k s g A$ mutants fail to dimethylate the 16S rRNA and, in consequence, are resistant to kasugamycin (an antibiotic related to the aminoglycoside family). The nondimethylated ribosomal 
particles are mildly impaired in protein synthesis, mainly at the initiation and elongation steps, and $k s g A$ mutants show a slight decrease in growth rate.

In eukaryotes, the functional role of this dimethylation, and indeed all other rRNA modifications, is unknown. The putative yeast $18 \mathrm{~S}$ rRNA dimethylase gene, DIM1, was cloned by complementation of the kasugamycin sensitivity of the $E$. coli $k s g A$ mutant. Dimlp is clearly homologous to KsgAp and can dimethylate the $E$. coli 16S rRNA in vivo (Lafontaine et al. 1994). In contrast to $E$. coli $k s g A$, yeast DIM1 is essential for viability, and we therefore generated a conditional allele to determine whether Dim 1p is required for ribosome synthesis or function.

\section{Results}

\section{Subcellular localization of the dimethylation event}

The presence of the $\mathrm{m}_{2}^{6} \mathrm{Am}_{2}^{6} \mathrm{~A}$ methylation can be detected by primer extension because the modification prevents normal base-pairing and therefore blocks reverse transcriptase. In Figure 2 this is shown by primer extension on pre-rRNA transcribed from the pGAL::rDNA plasmid (Henry et al. 1994) that contains the entire prerRNA coding region placed under the control of an RNA polymerase II promoter. This was expressed in an rpa12 mutant strain, which is thermosensitive for RNA Pol I (Nogi et al. 1993), allowing transcription of the chromosomal rDNA to be inhibited. On wild-type pre-rRNA, the methylation can be detected using oligonucleotides that hybridize within the $20 \mathrm{~S}$ pre-rRNA /e.g., oligonu-

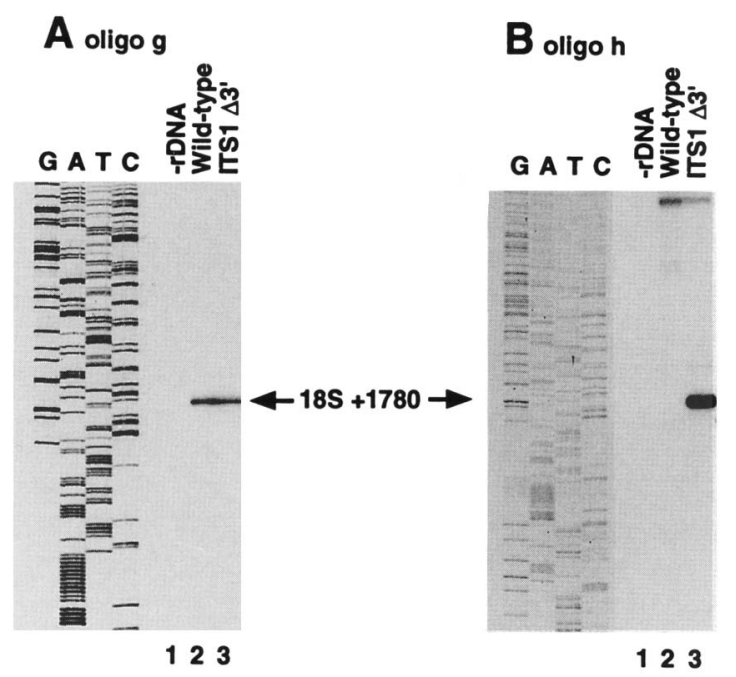

Figure 2. Primer extension through the dimethylation site using oligonucleotides $\mathrm{g}$ and $\mathrm{h}$, which hybridize to sequences in ITS1 $5^{\prime}$ and $3^{\prime}$ to site A2, respectively (Fig. 1). RNA was extracted from rpa12 strains carrying pGAL::rDNA containing the wild-type rDNA (wild-type lanes), pGAL:: rDNA containing a deletion of the $3^{\prime}$ region of ITS1 (ITS1 $\Delta 3^{\prime}$ lanes), or the same vector lacking the rDNA (-rDNA lanes), following growth at $37^{\circ} \mathrm{C}$ for $6 \mathrm{hr}$. DNA sequencing reactions on the wild-type rDNA plasmid using the same primers are also shown. cleotide g; see Fig. 1) but not using oligonucleotides that hybridize further $3^{\prime}$ (e.g., oligonucleotides $h-m$; see Fig. 1). This is shown for oligonucleotides $g$ and $h$ in Fig. 2 (wild-type lanes); these oligonucleotides hybridize to sequences in ITS1 that are separated only by 13 nucleotides, but cleavage site A2, the $3^{\prime}$ end of the $20 \mathrm{~S}$ prerRNA, lies in this interval. This is consistent with previous reports (Klootwijk et al. 1972; Salim and Maden 1973; Brand et al. 1977) that methylation is a late maturation event occurring on the $20 \mathrm{~S}$ pre-rRNA prior to its conversion to $18 \mathrm{~S}$ rRNA. Deletion of the $3^{\prime}$ region of ITS 1 can delay pre-rRNA processing and synthesis of $20 \mathrm{~S}$ pre-rRNA (Henry et al. 1994). On such mutant prerRNA, primer extension stops resulting from the $\mathrm{m}_{2}^{6} \mathrm{Am} \mathrm{m}_{2}^{6} \mathrm{~A}$ methylation can be observed with oligonucleotides $h-m$ (shown for oligonucleotide $h$ in Fig. 2, ITS $1 \Delta 3^{\prime}$ lanes), demonstrating that the $32 S$ pre-rRNA, or larger precursors, is methylated. Similarly, mutations in RRP2 (NME1) or POP1, which encode the RNA and protein components of RNase MRP, respectively, also delay synthesis of 20S pre-rRNA (Lygerou et al. 1994) and lead to methylation of the $32 \mathrm{~S}$ pre-rRNA (data not shown). Because the processing of $32 \mathrm{~S}$ to $20 \mathrm{~S}$ pre-rRNA is nucleolar, methylation can occur in the nucleolus.

\section{Construction of a conditional DIM1 allele}

To investigate the function of Dimlp, a conditional allele was created. The DIM1 promoter was replaced in vitro by the GAL10 promoter, and the GAL10::dim1 construct was used to replace the chromosomal DIM1 gene in a haploid yeast strain (see Materials and methods). Genes under the control of a $G A L$ promoter are expressed when the cells are grown on medium containing galactose and repressed by growth on glucose (Johnston and Davis 1984). This allows Dimlp to be depleted from the cells by growth following a shift from galactose to glucose medium. Northern hybridization demonstrated that the level of the DIM1 mRNA falls rapidly after transfer of the $G A L:: \operatorname{dim} 1$ strain to glucose medium (data not shown). Experiments shown in Figures $3,4,5$, and 7, below, compare the GAL::dim1 strain with the otherwise isogenic parental DIM1 strain.

The GAL::dim1 strain was pregrown on galactose minimal medium to mid-log phase and transferred to glucose minimal medium. For approximately six generations after transfer to glucose, the $G A L:: \operatorname{dim} 1$ strain grows identically to the wild-type strain, doubling every $2.5 \mathrm{hr}$. From $15 \mathrm{hr}$ on, there is a progressive increase in the doubling time of the GAL::dim1 strain, whereas the wild type maintains a constant doubling time of $2.5 \mathrm{hr}$. After $33 \mathrm{hr}$ of growth on glucose, the GAL::dim 1 strain has a doubling time of $5.1 \mathrm{hr}$. Repression of the $G A L:: \operatorname{dim} 1$ construct is probably leaky, allowing residual Dimlp synthesis; this is also the case for many other genes expressed under the control of $G A L$ promoters.

To test for depletion of the dimethylation activity, primer extension was performed through the site of dimethylation (Fig. 3) using a primer that hybridizes within the $20 \mathrm{~S}$ pre-rRNA (oligonucleotide $\mathrm{g}$ in Fig. 1). 


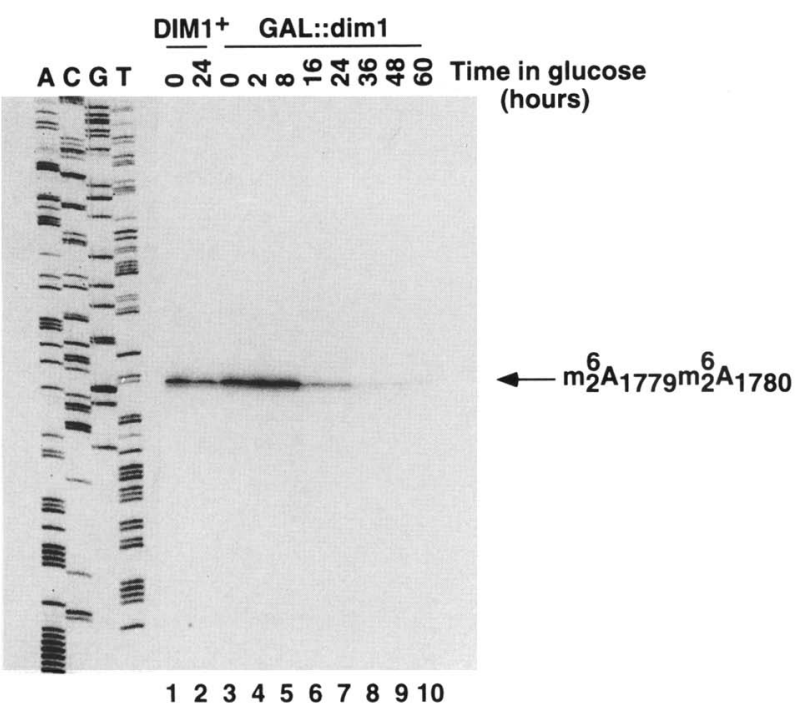

Figure 3. Primer extension through the dimethylation site. Primer extension was performed using oligonucleotide g, which hybridizes to the $20 \mathrm{~S}$ pre-rRNA within ITS1 (Fig. 1). (Lane 1) RNA extracted from the wild-type strain following growth on galactose medium; (lane 2) RNA extracted from the wild-type strain following growth on glucose medium for $24 \mathrm{hr}$; (lane 3) RNA extracted from the $G A L:: \operatorname{dim} 1$ strain following growth on galactose medium; (lanes 4-10) RNA extracted from the $G A L:: \operatorname{dim} 1$ strain following growth on glucose medium for 2, 8, $16,24,36,48$, and $60 \mathrm{hr}$, respectively. RNA was extracted from equal numbers of cells, as judged by $\mathrm{OD}_{600}$ units. The positions of primer extension stops resulting from the presence of the modification are indicated. DNA sequencing reactions using oligonucleotide $\mathrm{g}$ are also shown.

Total RNA was extracted from equivalent numbers of cells from the wild-type $\left(D I M 1^{+}\right)$and $G A L:: \operatorname{dim} 1$ strains grown on galactose $(0 \mathrm{hr})$ and at intervals after transfer to glucose medium. In the GAL::dim1 strain, the level of dimethylated pre-rRNA species decreases during growth on glucose medium, the largest drop occurring between 8 and $16 \mathrm{hr}$ following transfer (Fig. 3). These data indicate that Dimlp is responsible for making the $\mathrm{m}_{2}^{6} \mathrm{~A}_{1779} \mathrm{~m}_{2}^{6} \mathrm{~A}_{1780}$ dimethylation.

Even at late time points, the $\mathrm{m}_{2}^{6} \mathrm{~A}_{1779} \mathrm{~m}_{2}^{6} \mathrm{~A}_{1780}$ primer extension stop does not completely disappear, consistent with a lack of complete depletion of Dim1p. The same RNA preparations were used for the experiments shown in Figures 3, 4, 5, and 7A; the data presented below do not indicate that the total level of pre-rRNA species that contain the site of methylation and the site of priming is greatly reduced in strains depleted of Dimlp. The decrease in methylated pre-rRNA is therefore not the result of rapid degradation of the pre-rRNA.

\section{Effects of Dim1p depletion on pre-rRNA processing}

The steady-state levels of the mature rRNAs and prerRNAs during the time course of Dimlp depletion were analyzed by Northern hybridization (Fig. 4A-F). The level of $18 \mathrm{~S}$ rRNA falls during depletion, whereas the 25S rRNA level remains constant (Fig. 4A).

The pre-rRNA processing pathway is shown in Figure 1. The $35 \mathrm{~S}$ pre-rRNA is initially cleaved at site $\mathrm{A} 0$ in the 5' ETS generating the 33S pre-rRNA; the 33S pre-rRNA is then cleaved at site $\mathrm{Al}$, the $5^{\prime}$ end of the mature $18 \mathrm{~S}$ rRNA sequence, generating the $32 \mathrm{~S}$ pre-rRNA. The $35 \mathrm{~S}$ rRNA is detected by all pre-rRNA probes (Fig. 4B-F); the level of the $35 \mathrm{~S}$ pre-rRNA is little affected in the GAL:: $\operatorname{dim} 1$ strain. A probe specific for the 5 ' ETS sequence present in $33 \mathrm{~S}$ but not on $32 \mathrm{~S}$ (Fig. $4 \mathrm{C}$ ) reveals that in the GAL::dim1 strain the 33S pre-rRNA accumulates strongly, commencing $16 \mathrm{hr}$ after transfer to glucose medium. The $33 \mathrm{~S}$ and $32 \mathrm{~S}$ pre-rRNAs are very similar in size and are not well resolved by Northern hybridization; however, close inspection reveals the mobility shift indicative of a change from $32 \mathrm{~S}$ to $33 \mathrm{~S}$ at $16 \mathrm{hr}$ after transfer to glucose (e.g., see Fig. 4B, lanes 5,6). Comparison of the signal in panels in which both $33 \mathrm{~S}$ and $32 \mathrm{~S}$ are detected (Fig. 4B,D-F) with the 33S-specific signal (Fig. $4 \mathrm{C}$ ) are also consistent with a reduction in the level of $32 \mathrm{~S}$ from 16 hours after transfer. We conclude that $33 \mathrm{~S}$ pre-rRNA is accumulated whereas $32 \mathrm{~S}$ pre-rRNA is depleted and therefore that processing at site $\mathrm{A} 0$ is not affected whereas processing at Al is inhibited.

In the wild type, the $32 \mathrm{~S}$ pre-rRNA is cleaved at site A2 in ITS1, generating the 20S and 27SA pre-rRNAs. Both the 20S pre-rRNA (Fig. 4D) and 27SA (Fig. 4E,F) pre-rRNAs are underaccumulated in the $G A L:: \operatorname{dim} 1$ strain, with the pre-rRNA levels dropping substantially between 8 and $16 \mathrm{hr}$ after transfer to glucose medium. In addition, a novel $22 \mathrm{~S}$ pre-rRNA species is detected in the $G A L:: \operatorname{dim} 1$ strain. This species is detected with probes to the region between $\mathrm{A} 0$ and $\mathrm{A} 1$ (probes $\mathrm{b}$ and $\mathrm{c}$ in Fig. 1 ; shown for probe $\mathrm{c}$ in Fig. $4 \mathrm{C}$ ) and between $\mathrm{A} 2$ and $\mathrm{A} 3$ (probe $\mathrm{h}$ in Fig. 1; Fig. 4E) but is not detected with a probe $5^{\prime}$ to site A0 (probe a in Fig. 1; data not shown) or $3^{\prime}$ to site A3 (probe $i$ in Fig. 1; Fig. 4F). From its size and hybridization pattern, the $22 \mathrm{~S}$ pre-rRNA is likely to extend from site $A 0$ to site $A 3$. This is the expected product if pre-rRNA processing is inhibited at sites $\mathrm{Al}$ and $\mathrm{A} 2$ but continues at sites $\mathrm{A} 0$ and $\mathrm{A} 3$.

In contrast to the pathway of $18 \mathrm{~S}$ rRNA synthesis, the $27 \mathrm{SB}$ precursor, which is specific to the processing pathway leading to the $5.8 \mathrm{~S}$ and $25 \mathrm{~S}$ rRNAs, is not affected by depletion of Dimlp (Fig. 4B). The 27SA' pre-rRNA cannot be detected by Northern hybridization, but primer extension through site $\mathrm{A} 3$, the $5^{\prime}$ end of $27 \mathrm{SA}^{\prime}$, shows that its accumulation is unaffected in the GAL:: $\operatorname{dim} 1$ strain (data not shown). The levels of the 5.8S rRNA and its $7 \mathrm{~S}$ precursor are also unaffected in the GAL::dim1 strain, and no alteration was detected in the relative use of the alternative processing pathways that lead to the formation of the long and short forms of 5.8S rRNA (Henry et al. 1994, Lygerou et al. 1994) (data not shown).

Genetic depletion of the RNA or protein components of the U3, U14, snR10, or snR30 small nucleolar ribonucleoproteins (snoRNPs) also inhibits processing at sites $\mathrm{A} 1$ and A2, preventing synthesis of the mature $18 \mathrm{~S}$ rRNA (Tollervey 1987; Li et al. 1990; Hughes and Ares 
A.

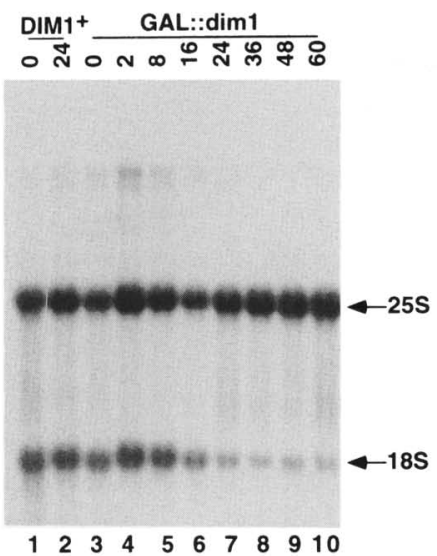

D.

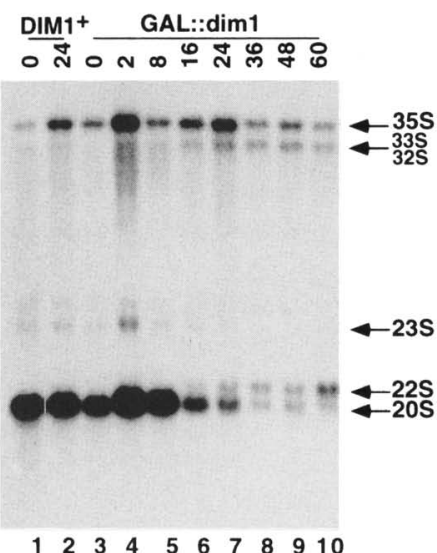

B.

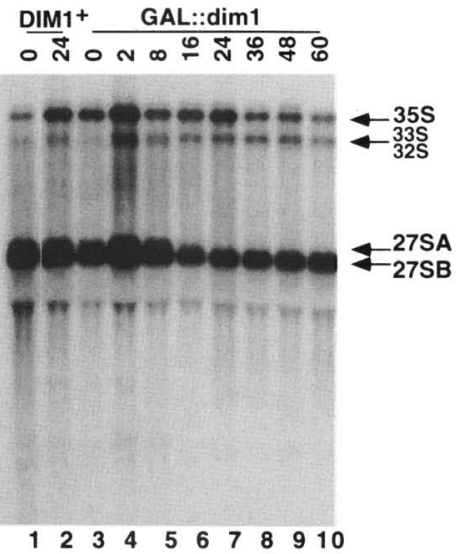

E.

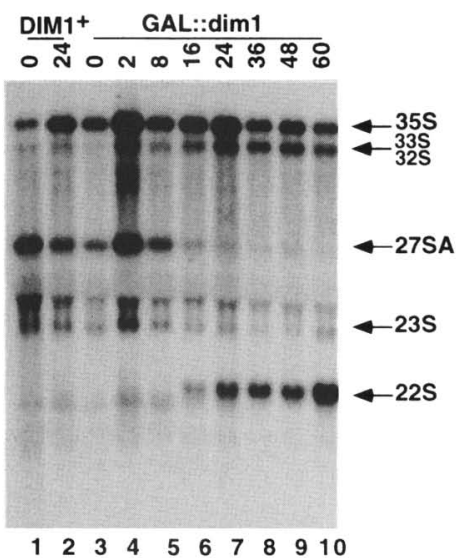

C.

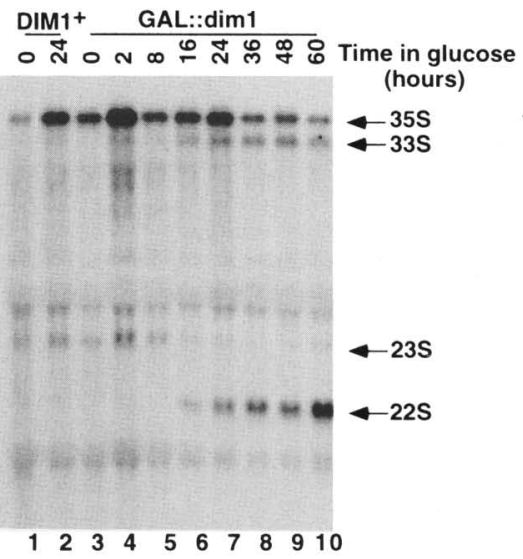

F.

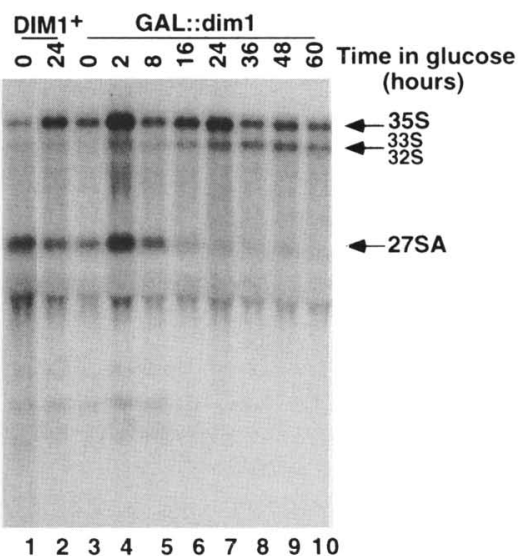

Figure 4. Steady-state levels of mature and precursor rRNA. (Lane 1) RNA extracted from the wild-type strain following growth on galactose medium; (lane 2) RNA extracted from the wild-type strain following growth on glucose medium for $24 \mathrm{hr}$; (lane 3) RNA extracted from the GAL::dim1 strain following growth on galactose medium; (lanes 4-10) RNA extracted from the GAL::dim1 strain following growth on glucose medium for $2,8,16,24,36,48$, and $60 \mathrm{hr}$, respectively. (A) Hybridization with oligonucleotides complementary to the mature $18 \mathrm{~S}$ and $25 \mathrm{~S}$ IRNAs (oligonucleotides $\mathrm{d}$ and 1$)$. (B) Hybridization with an oligonucleotide complementary to the $5^{\prime}$ region of ITS2 (oligonucleotide $\mathrm{k}$ ). (C) Hybridization with an oligonucleotide complementary to the $3^{\prime}$ region of the $5^{\prime}$ ETS, between cleavage site $\mathrm{A} 0$ and $\mathrm{A} 1$ (oligonucleotide $\mathrm{c}$ ). (D) Hybridization with an oligonucleotide complementary to the $5^{\prime}$ region of ITS1, upstream of cleavage site A2 (oligonucleotide g). $(E)$ Hybridization with an oligonucleotide complementary to the central region of ITS1, between cleavage sites A2 and A3 (oligonucleotide h). (F) Hybridization with an oligonucleotide complementary to the 3' region of ITS1, downstream of cleavage site A3 (oligonucleotide i). RNA was extracted from equal numbers of cells, as judged by $\mathrm{OD}_{600}$ units. The positions of the mature rRNAs and the pre-rRNAs are indicated; the 33S and 32S pre-rRNAs are not well resolved. The positions of the oligonucleotides used are indicated in Fig. 1.

1991; Tollervey et al. 1991; Girard et al. 1992; Jansen et al. 1993; Morrissey and Tollervey 1993). The pattern of processing observed in these mutants is, however, different from that seen in the Dimlp-depleted strain; no accumulation of the $33 \mathrm{~S}$ or $22 \mathrm{~S}$ pre-rRNAs is observed, instead the snoRNP mutants accumulate a $23 \mathrm{~S}$ prerRNA that extends from the $5^{\prime}$ end of the 35 S pre-rRNA to site A3 and is generated by direct cleavage of the $35 \mathrm{~S}$ pre-rRNA at A3 (Henry et al. 1994). The 23S pre-rRNA can be detected at a low level in wild-type strains (Figs. $4 C, D$, lanes 1,2 ) but does not increase in the $G A L:: \operatorname{dim} 1$ strain during growth on glucose medium.

The kinetics of the inhibition of pre-rRNA processing and pre-rRNA methylation during depletion of Dimlp are in close agreement; compare the loss of $20 \mathrm{~S}$ prerRNA in Figure 4D with the loss of the primer extension stop resulting from the $\mathrm{m}_{2}^{6} \mathrm{~A}_{1779} \mathrm{~m}_{2}^{6} \mathrm{~A}_{1780}$ modification in Figure 3.

Primer extension was used to determine whether the $33 \mathrm{~S}$ and $22 \mathrm{~S}$ pre-rRNA species that accumulate in the GAL:: dim 1 strain have $5^{\prime}$ ends at site $\mathrm{A} 0$ or are the result of processing at an aberrant site (Fig. 5). Strong accumulation of pre-rRNA species with 5' ends at site A0 is seen in the GAL::dim1 strain during growth on glucose medium. The time course of accumulation is in good agreement with the accumulation of the $33 \mathrm{~S}$ and $22 \mathrm{~S}$ pre- 


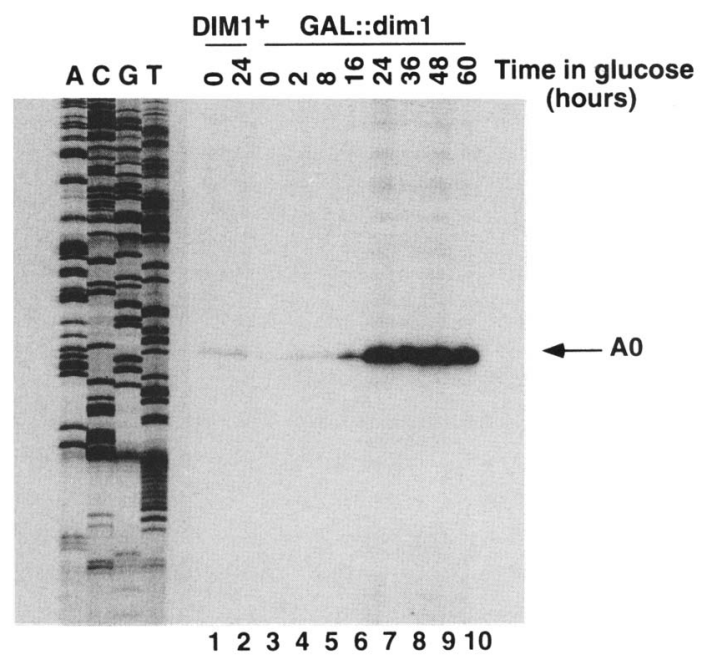

Figure 5. Primer extension in the $5^{\prime}$ ETS. Primer extension was performed using oligonucleotide $\mathrm{d}$, which hybridizes within the $5^{\prime}$ region of the $18 \mathrm{~S}$ rRNA (Fig. 1). (Lane 1) RNA extracted from the wild-type strain following growth on galactose medium; (lane 2) RNA extracted from the wild-type strain following growth on glucose medium for $24 \mathrm{hr}$; (lane 3) RNA extracted from the GAL::dim1 strain following growth on galactose medium; (lanes 4-10) RNA extracted from the GAL::dim1 strain following growth on glucose medium for 2,8 , $16,24,36,48$, and $60 \mathrm{hr}$, respectively. The position of the primer extension stop as a result of the cleavage of the pre-rRNA at site $\mathrm{AO}$ is indicated. DNA sequencing reactions using oligonucleotide $\mathrm{d}$ are also shown.

rRNAs detected by Northern hybridization. Primer extension also shows that the residual processing at sites $\mathrm{Al}$ and $\mathrm{A} 2$ is correct at the nucleotide level at all time points in the GAL::dim1 strain, as is processing at sites $\mathrm{A} 3, \mathrm{~B} 1(\mathrm{~L})$, and $\mathrm{B} 1(\mathrm{~S})$ (data not shown).

We conclude that in the GAL::dim1 strain, cleavage of the pre-rRNA is specifically inhibited at sites $\mathrm{A} 1$ and $\mathrm{A} 2$, the $5^{\prime}$ and $3^{\prime}$ ends of the 20 s pre-rRNA, respectively. This would prevent the synthesis of the 20S pre-rRNA and, therefore, of the mature $18 \mathrm{~S}$ rRNA. Upstream processing of the $35 \mathrm{~S}$ primary transcript at site $\mathrm{A} 0$ in the $5^{\prime}$ ETS is not affected nor are subsequent processing steps on the pathway of synthesis of $5.8 \mathrm{~S}$ and $25 \mathrm{~S}$ rRNA.

To confirm that mature 18S rRNA is synthesized with a reduced efficiency in the $G A L:: \operatorname{dim} 1$ strain, a metabolic labeling experiment was performed (Fig. 6). Following $25 \mathrm{hr}$ of growth in glucose medium, cells were pulselabeled for $1 \mathrm{~min}$ with $\left[{ }^{3} \mathrm{H}\right]$ uracil and then chased with a large excess of unlabeled uracil for the times indicated. Total incorporation of $\left[{ }^{3} \mathrm{H}\right]$ uracil into rRNA in the mutant strain is lower than in the wild type, presumably because of the effects of the reduced growth rate on prerRNA transcription. Comparison of the ratio of $18 \mathrm{~S}-25 \mathrm{~S}$ rRNA synthesis makes it clear, however, that $18 \mathrm{~S}$ rRNA is underaccumulated in the GAL::dim1 strain (cf. lanes 4 and 8). Synthesis the of 27SA and 20S pre-rRNAs is also strongly reduced in the $G A L:: \operatorname{dim} 1$ strain. The $22 \mathrm{~S}$ prerRNA species is readily detected (Fig. 6, lanes 7,8), dem- onstrating that it is a major processing product in the Dimlp-depleted strain, rather than being synthesized at low levels and accumulating in the mutant. The residual $18 \mathrm{~S}$ rRNA that is synthesized in the mutant does not appear to be less stable than in the wild type because it is resistant to chasing for a period of $60 \mathrm{~min}$ (data not shown).

Even at late time points after transfer of the GAL::dim1 strain to glucose medium, the 20S (Fig. 4D) and 27SA (Fig. 4E,F) pre-rRNAs can be detected, showing that some residual processing at sites $\mathrm{A} 1$ and $\mathrm{A} 2$ continues. The question therefore arises whether this is the result of the processing of the residual methylated prerRNA species or whether nonmethylated pre-rRNAs can also be processed, resulting in the synthesis of nonmethylated $18 \mathrm{~S}$ rRNA. As described above, primer extension through site $\mathrm{A}_{1779} \mathrm{~A}_{1780}$ can be specifically blocked by the presence of the dimethyl groups. This was used to determine whether the residual 18S rRNA which is synthesized in the mutant is dimethylated. To assess the degree of methylation, primer extension was carried out from oligonucleotide $f$, complementary to the $3^{\prime}$ end of $18 \mathrm{~S}$ rRNA (Figs. 1 and $7 \mathrm{C}$ ) using dideoxyadenosine nucleotides in the reaction in place of deoxyadenosine. On nondimethylated rRNA, reverse transcriptase extends through the $\mathrm{A}_{1779} \mathrm{~A}_{1780}$ site and is specifically blocked two nucleotides further $5^{\prime}$, at $U_{1777}$, owing to the incorporation of dideoxyadenosine into the cDNA. The ratio of readthrough (product $c_{;}$Fig. 7) to $\mathrm{m}_{2}^{6} \mathrm{~A}$ terminated product (products $\mathrm{a}+\mathrm{b}$ ) is a measure of the level of nonmodified versus modified 18S rRNA.

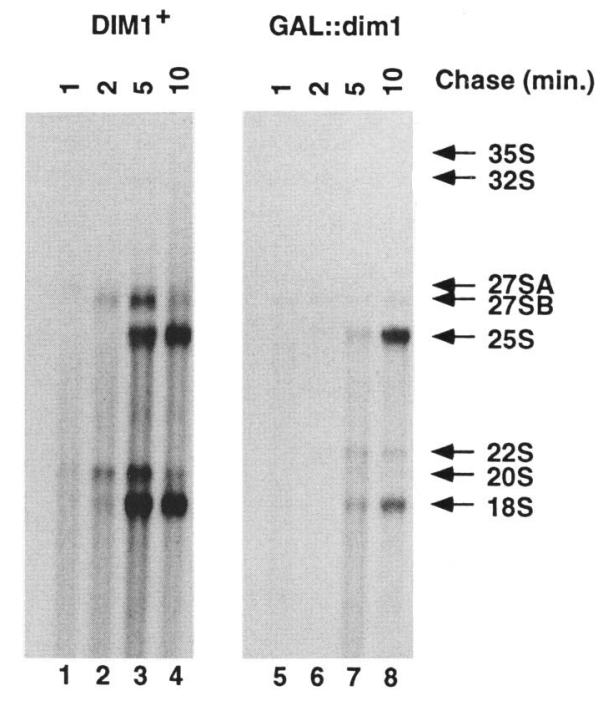

Figure 6. Metabolic labeling of pre-rRNA from wild-type $\left(D I M 1^{+}\right)$and $G A L:: \operatorname{dim} 1$ strains following growth on glucose medium for 25 he. (Lanes 1-4) RNA extracted from the wildtype strain; (lanes 5-8) RNA extracted from the GAL::dim1 strain. Cells were pulse-labeled with $\left[{ }^{3} \mathrm{H}\right]$ uracil for $1 \mathrm{~min}$ at $30^{\circ} \mathrm{C}$. A large excess of unlabeled uracil was then added, and the cells were incubated for $1,2,5$, and $10 \mathrm{~min}$ as indicated. The positions of the mature rRNAs and the major pre-rRNAs are indicated. 


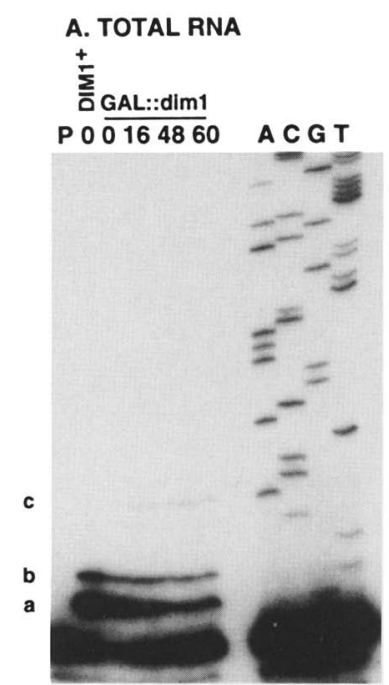

123456

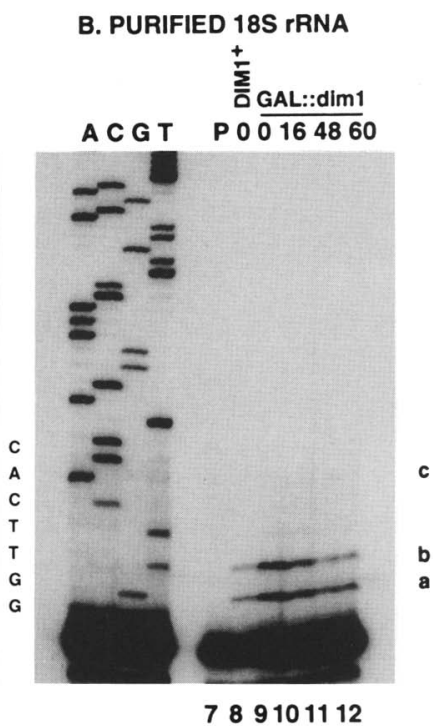

c.

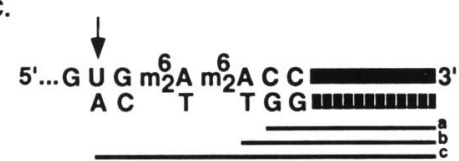

Figure 7. Primer extension through the site of dimethylation. Primer extension was performed with oligonucleotide $f$, complementary to the $3^{\prime}$ end of 18S rRNA (Fig. 1) and using only dideoxyadenosine nucleotides in place of deoxyadenosine. The site of priming is 3 nucleotides $3^{\prime}$ to $A_{1780}$, and no A residues are incorporated before the site of modification. Dimethylation of $\mathrm{A}_{1779} \mathrm{~A}_{1780}$ blocks primer extension at that site. Extensions carried out on nondimethylated rRNA extend through the $\mathrm{A}_{1779} \mathrm{~A}_{1780}$ site but are blocked 2 nucleotides $5^{\prime}$ to $\mathrm{A}_{1779}$ (at position $\left.\mathrm{U}_{1777}\right)$. (A) Total RNA. (B) Purified 18S rRNA. The same amount of total RNA or gel-purified 18S rRNA was used for each lane. (Lanes 1,7) No RNA, symbol P denotes primer alone; (lanes 2,8) RNA extracted from the wild-type strain following growth on galactose medium; (lanes 3,9) RNA extracted from the GAL::dim1 strain following growth on galactose medium; (lanes 4-6,10-12) RNA extracted from the GAL::dim1 strain following growth on glucose medium for 16,48 , and 60 $h r$, respectively. (C) Schematic representation of the 3 ' end of $18 \mathrm{~S}$ rRNA. (Upper line) rRNA strand; the thick line represents the last 16 nucleotides. (Lower line) Complementary cDNA strand; the thick broken line represents oligonucleotide $f$. The three potential extended products are represented by thin lines (labeled a, b, and c). The positions of the primer extension stops resulting from the presence of the modification are indicated as $a$ and $b$; the position of the primer extension stop resulting from readthrough of the dimethylation site is indicated as c. DNA sequencing reactions using oligonucleotide $f$ are shown, and the nucleotide sequence, corresponding to the cDNA strand /lower lane of $C$ ), is indicated between $A$ and $B$. It is not clear whether the stop identified as $\mathrm{b}$ is a consequence of incomplete dimethylation or is a primer extension artifact.

When this experiment is carried out on standardized amounts of purified 18S rRNA (Fig. 7B), the readthrough is not detected. On total RNA (Fig. 7A) some read- through is expected owing to newly transcribed prerRNA molecules that are still nondimethylated (Fig. 7A, lanes 2,3$)$. Following transfer of the GAL::dim1 strain to glucose medium, the level of readthrough increases (Fig. 7A, lanes 4-6), presumably as a result of the accumulation of nonmethylated $33 \mathrm{~S}$ and $22 \mathrm{~S}$ pre-rRNA, whereas the $\mathrm{m}_{2}^{6} \mathrm{~A}$ terminated product declines as a result of the reduced accumulation of mature $18 \mathrm{~S}$ rRNA.

Nondimethylated $18 \mathrm{~S}$ rRNA is not detected even 60 hours (>12 generations) after transfer of the GAL::dim1 strain to glucose medium; if the $18 \mathrm{~S}$ rRNA synthesized after Dimlp depletion were nondimethylated, it would have been expected to constitute a major fraction of the rRNA by this time. The low level of $18 \mathrm{~S}$ rRNA observed at late time points by Northern hybridization (Fig. 4A, lanes 8-10) and pulse-chase labeling (Fig. 6, lanes 7,8) can therefore be attributed to processing of residual, methylated $20 S$ pre-rRNA. Although we cannot exclude the possibility that some level of unmethylated $18 \mathrm{~S}$ rRNA is synthesized and immediately degraded, our data strongly indicates that only correctly methylated pre-rRNA species are processed. This could be due to a requirement for the formation of the $\mathrm{m}_{2}^{6} \mathrm{Am}_{2}^{6} \mathrm{~A}$ residues or to a requirement for an association of Dimlp with the pre-rRNA; the absence of Dim $1 \mathrm{p}$ binding might prevent both processing and methylation.

\section{Construction of a cis-acting $G_{1779} G_{1780}$ mutant}

To test whether formation of the dimethyladenines is required for pre-rRNA processing, a pre-rRNA mutation was created in which the twin adenosines $A_{1779} A_{1780}$ were exchanged for guanosine residues. To assess its effect on ribosome biogenesis, the $G_{1779} G_{1780}$ mutation was expressed from plasmid pGAL::rDNA (Henry et al. 1994) in an rpa12 strain (Nogi et al. 1993). As described above, chromosomal rDNA transcription is inhibited at the nonpermissive temperature $\left(37^{\circ} \mathrm{C}\right)$, allowing analysis of the processing of the mutant pre-rRNA. The low level of residual chromosomal transcription does not interfere with the identification of pre-rRNA produced from the pGAL::rDNA plasmid. The presence of neutral tags in the $18 \mathrm{~S}, 5.8 \mathrm{~S}$, and $25 \mathrm{~S}$ rDNA sequences in pGAL::rDNA also allows the identification of mature rRNAs synthesized from the mutant pre-rRNA at all temperatures (Beltrame et al. 1994; Henry et al. 1994).

The absence of modification in the mutant pre-rRNA was confirmed by primer extension (Fig. 8). The guanosine residues should not be a substrate for $N^{6}, N^{6}$ dimethylation, and no methylation of the mutant prerRNA is detected. The same RNA preparations were used for the experiments shown in Figures 10 and 11, below, demonstrating that the pre-rRNAs are present, and on longer exposures, a similar level of background primer extension stops is seen in all lanes /data not shown).

\section{Effects of the $G_{1779} G_{1780}$ mutation on $r R N A$ processing}

The pGAL::rDNA plasmid carrying the wild-type rDNA 
supports growth of the rpa12 strain at $37^{\circ} \mathrm{C}$ (Fig. 9, wildtype sectors). In contrast, the same plasmid carrying the rDNA with $\mathrm{G}_{1779} \mathrm{G}_{1780}$ mutation (Fig. 9, $\mathrm{G}_{1779} \mathrm{G}_{1780}$ sectors) or lacking the rDNA (Fig. 9, -rDNA sectors) does not support growth of the rpa12 strain at $37^{\circ} \mathrm{C}$. At $25^{\circ} \mathrm{C}$, all strains can grow because the chromosomal rDNA is transcribed. We conclude that this mutation prevents the synthesis of functional ribosomes.

The effects of the $\mathrm{G}_{1779} \mathrm{G}_{1780}$ mutation on the steadystate levels of mature rRNA and of pre-rRNA were analyzed $6 \mathrm{hr}$ after transfer to $37^{\circ} \mathrm{C}$. RNA was extracted, run on an agarose-formaldehyde gel, and transferred for Northern hybridization with a set of probes specific for various regions of the $35 \mathrm{~S}$ pre-rRNA (Fig. 10A-F). No processing defect is seen in the $\mathrm{G}_{1779} \mathrm{G}_{1780}$ mutant (Fig. 10A-F). No $33 \mathrm{~S}$ pre-rRNA accumulation is observed, 27SA and 20S pre-rRNA, as well as mature 18S rRNA, are synthesized normally, and the aberrant $22 \mathrm{~S}$ prerRNA observed upon Dimlp depletion is not detected.

Accumulation of $18 \mathrm{~S}$ rRNA carrying the $\mathrm{G}_{1779} \mathrm{G}_{1780}$ mutation is partially cold sensitive (Fig. 11). Northern hybridization of RNA extracted from cells grown at $23^{\circ} \mathrm{C}$ and hybridized with oligonucleotides complementary to the tags in the $18 \mathrm{~S}$ and $25 \mathrm{~S}$ rRNAs /oligonucleotides $\mathrm{e}$

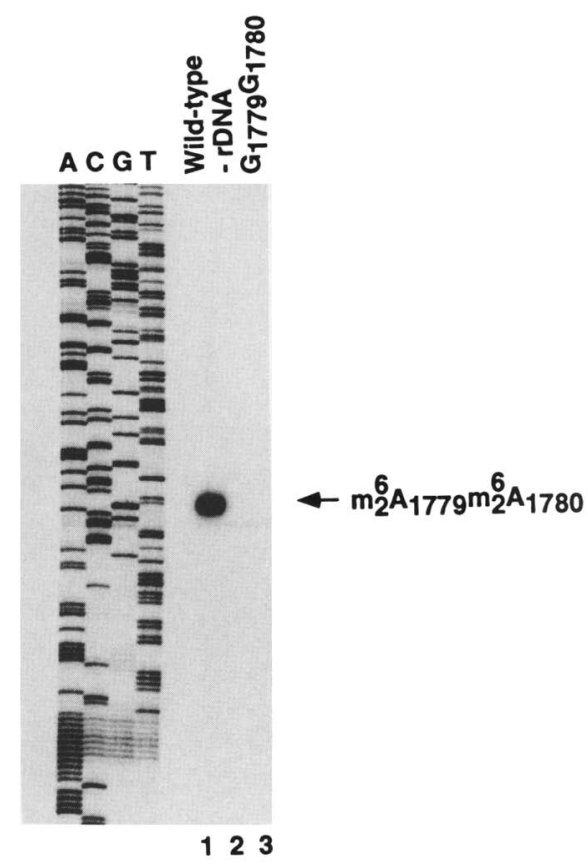

Figure 8. Primer extension through the dimethylation site in the $\mathrm{G}_{1779} \mathrm{G}_{1780}$ mutant. Primer extension was performed using oligonucleotide $\mathrm{g}$ (Fig. 1). (Lane 1) pGAL::rDNA containing the wild-type rDNA; (lane 2) the same vector lacking the rDNA sequence; (lane 3) pGAL::rDNA containing the $\mathrm{G}_{1779} \mathrm{G}_{1780} \mathrm{mu}-$ tation. RNA was extracted from rpa12 strains carrying the plasmids indicated $6 \mathrm{hr}$ after transfer to $37^{\circ} \mathrm{C}$. The same amount of RNA from each strain was used. The positions of primer extension stops resulting from the presence of the modification are indicated. DNA sequencing reactions using oligonucleotide $g$ are also shown.
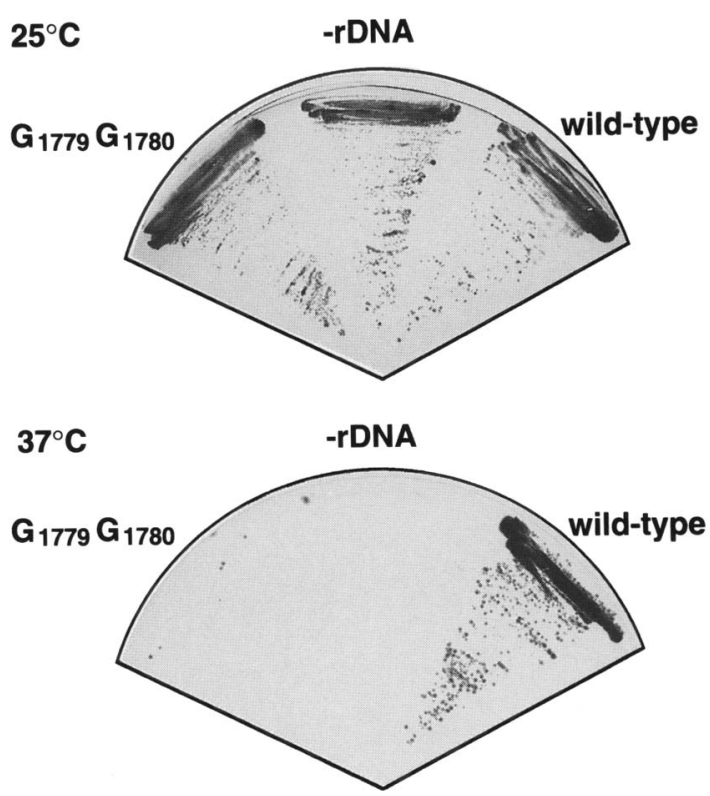

Figure 9. Pre-rRNA carrying the $\mathrm{G}_{1779} \mathrm{G}_{1780}$ mutation does not support growth. An rpa12 strain was transformed with pGAL: rDNA containing the wild-type rDNA (wild-type sectors) pGAL::rDNA containing the $\mathrm{G}_{1779} \mathrm{G}_{1780}$ mutation $\mid \mathrm{G}_{1779} \mathrm{G}_{1780}$ sectors), or with the same vector lacking the rDNA sequence (-rDNA sectors). Plates were incubated at $25^{\circ} \mathrm{C}$ (top) or $37^{\circ} \mathrm{C}$ (bottom).

and $\mathrm{m}$ ) reveals that the level of 18S rRNA falls drastically, whereas accumulation of $25 \mathrm{~S}$ rRNA is unaffected, (Fig. 11, cf. lanes 1 and 3). Growth at $18^{\circ} \mathrm{C}$ gives identical results (data not shown). The $\mathrm{G}_{1779} \mathrm{G}_{1780}$ mutation may be responsible for an assembly defect, enhanced at low temperature, leading to rapid 18S rRNA degradation. Even at $37^{\circ} \mathrm{C}$, the accumulation of $18 \mathrm{~S}$ rRNA is slightly lower for the $G_{1779} G_{1780}$ mutant than for the wild type (Fig. 10F, cf. lanes 1 and 3). As no processing defect is detected, this is consistent with increased instability of 18S rRNA molecules that are nondimethylated (Fig. 8) and that may be structurally altered by the $\mathrm{G}_{1779} \mathrm{G}_{1780}$ replacement. Experiments presented for Dimlp depletion in Figures 3-7 were performed on cells grown at $30^{\circ} \mathrm{C}$; depletion of Dimlp at $37^{\circ} \mathrm{C}$ resulted in an identical processing defect (data not shown).

\section{Discussion}

DIM1 is involved in pre-rRNA processing

The DIM1 gene was cloned by complementation of a mutation in the $E$. coli $k s g A$ dimethylase, which prevents the 16S rRNA dimethylation. Dimlp is highly homologous to KsgAp and can restore both 16S rRNA dimethylation and kasugamycin sensitivity in $E$. coli (Lafontaine et al. 1994). This made it probable that Dimlp is the enzyme responsible for making the homologous dimethylation, $\mathrm{m}_{2}^{6} \mathrm{~A}_{1779} \mathrm{~m}_{2}^{6} \mathrm{~A}_{1780}$, in the yeast $18 \mathrm{~S}$ rRNA. We have investigated the function of Dimlp by 


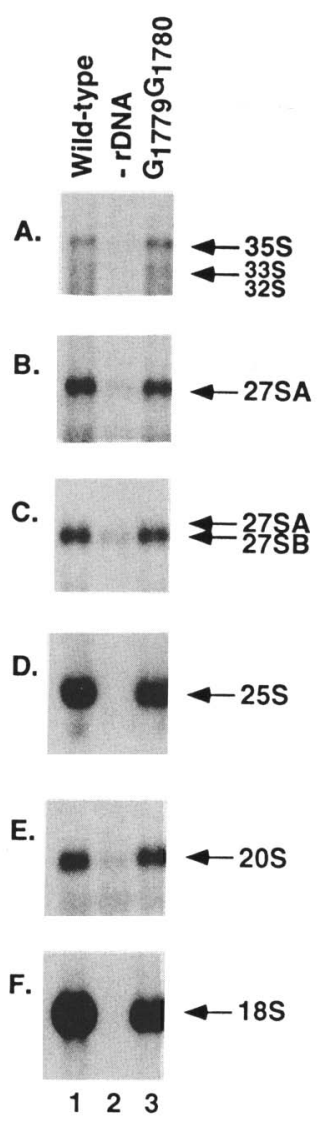

Figure 10. Northern analysis of pre-rRNA and rRNA from the $\mathrm{G}_{1779} \mathrm{G}_{1780}$ mutant at $37^{\circ} \mathrm{C}$. (Lane 1) pGAL::rDNA containing the wild-type rDNA; (lane 2) the same vector lacking the rDNA sequence; (lane 3) pGAL::rDNA containing the $\mathrm{G}_{1779} \mathrm{G}_{1780} \mathrm{mu}$ tation. RNA was extracted from rpa12 strains carrying the plasmids indicated $6 \mathrm{hr}$ after transfer to $37^{\circ} \mathrm{C}$. The same amount of RNA from each strain was used. $(A, B)$ Hybridization with an oligonucleotide complementary to ITS1 between cleavage sites $\mathrm{A} 2$ and $\mathrm{A} 3$ (oligonucleotide $\mathrm{h}$ ). (C) Hybridization with an oligonucleotide complementary to the $5^{\prime}$ region of ITS2 joligonucleotide $\mathrm{k}$. (D) Hybridization with an oligonucleotide complementary to the 25S rRNA tag (oligonucleotide $\mathrm{m}$ ). $(E)$ Hybridization with an oligonucleotide complementary to the 5 ' region of ITS1, upstream of cleavage site A2 (oligonucleotide g). $(F)$ Hybridization with an oligonucleotide complementary to the 18 S rRNA tag (oligonucleotide e). To simplify the figure, only the relevant regions of each Northern blot are shown; together the six panels present all the pre-rRNAs and rRNAs that we have detected. The positions of the oligonucleotides used are indicated in Fig. 1.

analysis of the effects of growth of a conditional GAL::dim1 mutant strain on glucose medium. Primer extension shows that the level of dimethylation of the pre-rRNA is progressively reduced during growth of the GAL::dim1 mutant on glucose medium, confirming that Dimlp is indeed responsible for this modification in yeast.

Analysis of pre-rRNA processing in the GAL::dim1 strain showed that, unexpectedly, Dimlp is specifically required for the cleavages that generate the $20 \mathrm{~S}$ prerRNA, the immediate precursor to the mature $18 \mathrm{~S}$ rRNA. Following transfer of the GAL::dim1 strain to glucose medium, processing is progressively inhibited at site $\mathrm{A} 1$, the $5^{\prime}$ end of both the $20 \mathrm{~S}$ pre-rRNA and the mature $18 \mathrm{~S}$ rRNA, and at site A2 in ITS1, the $3^{\prime}$ end of the $20 \mathrm{~S}$ pre-rRNA. In consequence, formation of the $18 \mathrm{~S}$ rRNA is strongly reduced. In contrast, we detect no requirement for Dimlp in the cleavage of the 5' ETS at site A0 nor in the subsequent processing reactions that generate the large subunit rRNA species, $5.8 \mathrm{~S}$ and $25 \mathrm{~S}$ rRNA. The kinetics of the inhibition of the pre-rRNA processing reactions are in close agreement with the loss of pre-rRNA methylation, suggesting that the processing of nonmethylated pre-rRNA is inhibited.

Even $60 \mathrm{hr}$ (>12 generations) after transfer to glucose medium, we do not detect nonmethylated 18S rRNA in the GAL::dim1 strain. This shows that the $18 \mathrm{~S}$ rRNA accumulated in the mutant is processed from the residual methylated pre-rRNA. We conclude that following transfer of the GAL::dim1 strain to glucose medium there is insufficient Dimlp to methylate all pre-rRNA molecules; only those pre-rRNAs that get methylated are processed at sites $\mathrm{Al}$ and $\mathrm{A} 2$. It appears that a system has developed to specifically prevent the synthesis of the $20 \mathrm{~S}$ pre-rRNA, and therefore the 18S rRNA, in the absence of methylation. We know of no other examples of the regulation of RNA processing reactions in response to modification.

The simplest model for the inhibition of processing would have been that the $\mathrm{m}_{2}^{6} \mathrm{Am}_{2}^{6} \mathrm{~A}$ residues are required for some aspect of ribosome assembly. For example, the correct folding of the (pre)-rRNA or the binding of a ri-

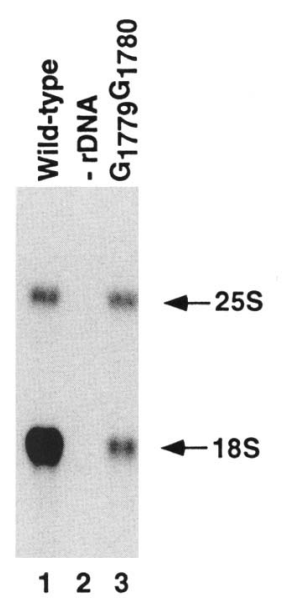

Figure 11. Northern analysis of high-molecular-weight rRNA synthesized from the $\mathrm{G}_{1779} \mathrm{G}_{1780}$ mutant grown at $23^{\circ} \mathrm{C}$. (Lane 1) pGAL::rDNA containing the wild-type rDNA; (lane 2) the same vector lacking the rDNA sequence; (lane 3) pGAL::rDNA containing the $\mathrm{G}_{1779} \mathrm{G}_{1780}$ mutation. RNA was extracted from rpa12 strains carrying the plasmids indicated following growth at $23^{\circ} \mathrm{C}$. The same amount of RNA from each strain was hybridized with oligonucleotides complementary to the tags in the $18 \mathrm{~S}$ and $25 \mathrm{~S}$ rRNAs (e and $\mathrm{m}$ in Fig. 1). 
bosomal protein might have been dependent on $\mathrm{m}_{2}^{6} \mathrm{Am}_{2}^{6} \mathrm{~A}$ formation. The alternative was that some system monitored either the binding of Dimlp itself or the disappearance of the nonmethylated A residues. To distinguish between these possibilities, we constructed and tested a mutation of the pre-rRNA in which both A residues at the site of methylation are replaced by $G$ residues. Processing of the pre-rRNA is unaffected by the double $\mathrm{G}$ mutation, demonstrating that the formation of the $\mathrm{m}_{2}^{6} \mathrm{Am}_{2}^{6} \mathrm{~A}$ residues is not required for processing of this pre-rRNA. Moreover, processing of the wild-type pre-rRNA predominantly occurs before the methylation reaction, and formation of the $\mathrm{m}_{2}^{6} \mathrm{Am}_{2}^{6} \mathrm{~A}$ residues presumably cannot, therefore, be required for processing. Together, these results make it most unlikely that formation of $\mathrm{m}_{2}^{6} \mathrm{Am} \mathrm{m}_{2}^{6} \mathrm{~A}$ is required for processing at sites $\mathrm{A} 1$ and $\mathrm{A} 2$.

Two general classes of model for the inhibition of prerRNA processing in Dimlp-depleted cells can be envisaged. Association of Dimlp with the pre-ribosomal particle might be required for processing; Dimlp might directly interact with components required for processing or be required for correct ribosome assembly. In either case, this could be mediated through the lysine-rich amino-terminal extension of Dimlp that is not present in the E. coli KsgAp (Lafontaine et al. 1994). An alternative possibility is that some factor could have a binding site that overlaps with that of Dimlp. Displacement of this factor by Dim $1 p$ binding might be required to allow processing. Such a factor could be an RNA or a protein, but it is notable that many of the snoRNA species identified in $S$. cerevisiae are not essential for viability and have no clear growth phenotype when their genes are disrupted. The functions of so many nonessential snoRNAs (10 have been reported to date) are not clear. It might be that some have roles in monitoring the correct assembly or modification of the pre-rRNA. Vertebrate U13 is complementary to the $A_{1779} A_{1780}$ loop region and is, therefore, a potential candidate for such a role. Based on genetic data, cleavage at sites $\mathrm{A} 1$ and $\mathrm{A} 2$ has been proposed to be coupled and to be carried out by a processing complex that involves multiple snoRNPs (for review, see Fournier and Maxwell 1993; Morrissey and Tollervey 1995). Whatever the actual mechanism involved, this processing complex is a likely target for the coordinated regulation of $\mathrm{A} 1 / \mathrm{A} 2$ processing.

\section{Role of the dimethylation in ribosome assembly or function}

The two adenosines that are the substrates for dimethylation, are required for ribosomal assembly or function. Ribosomes carrying the $\mathrm{G}_{1779} \mathrm{G}_{1780}$ mutation do not support growth, showing this mutation to be lethal. Furthermore, the $18 \mathrm{~S}$ rRNA level drastically falls at $23^{\circ} \mathrm{C}$, possibly reflecting an assembly defect, because these are often associated with cold sensitivity. It is not clear at present whether it is the absence of the dimethylation or of the universally conserved adenosine residues at these positions that is responsible for the growth defect and the cold-sensitive phenotype.

\section{Subcellular location of Dim1p and the dimethylation activity}

In wild-type yeast strains, dimethylation is not detected on precursors other than the 20S pre-rRNA. However, when synthesis of $20 \mathrm{~S}$ pre-rRNA is delayed by the inhibition of cleavage at site $\mathrm{A} 2$ as a result of the mutation of cis- or trans-acting factors, dimethylation of the $32 \mathrm{~S}$ prerRNA species is observed. The $32 \mathrm{~S}$ pre-rRNA is undoubtedly nucleolar, demonstrating that the dimethylation reaction can occur in the nucleolus. We have, moreover, shown here that Dimlp is required for nucleolar prerRNA processing reactions. It therefore seems clear that Dimlp can associate with the pre-rRNA in the nucleolus and can function there. On the basis of subcellular fractionation, it has been reported that the 20S pre-rRNA is processed to $18 \mathrm{~S}$ rRNA in the cytoplasm (Udem and Warner 1973) and that dimethylation occurs on the cytoplasmic 20S pre-rRNA (Klootwijk et al. 1972; Udem and Warner 1973; Brand et al. 1977). It remains possible that in wild-type cells Dimlp is transported to the cytoplasm in association with the 20S pre-rRNA before the dimethylation reaction occurs. The phenomenon of methylation of the $32 \mathrm{~S}$ pre-rRNA in mutants in which $20 \mathrm{~S}$ synthesis is delayed suggests that the timing of the actual dimethylation reaction is not dependent on the timing of pre-rRNA processing.

\section{Evolutionary implications}

There are a number of similarities between the processing of pre-rRNA in eukaryotes and bacteria. In both, processing on the $5^{\prime}$ and $3^{\prime}$ sides of the small-subunit rRNA is coupled. In bacteria this is achieved by direct basepairing between the 5 ' - and 3 '-flanking spacer sequences, whereas in eukaryotes, coupling has been proposed to be mediated by interactions in trans with the snoRNAs (for review, see by Morrissey and Tollervey 1995). It is possible that the more complex processing system in eukaryotes was, in part, selected because of the greater regulatory potential that it offers. In $E$. coli, inactivation of the KsgAp methylase leads to the synthesis of nonmethylated ribosomes. In contrast, it appears that the association of Dimlp with nucleolar pre-rRNA is monitored in yeast cells by a system that is independent of its dimethylase activity. We postulate that failure of binding activates a regulatory system that inhibits processing and thereby prevents the synthesis of unmodified rRNA. It may have been the potential for just this type of regulatory system that drove selection for the snoRNAdependent pre-rRNA processing system of eukaryotes.

\section{Materials and methods}

Strains and media

Standard $S$. cerevisiae growth and handling techniques were used. Transformation was by the PEG method of Klebe et al. 
(1983). The haploid DIM1+ strain is BWG1-7A (MATa, ura3-52, leu2-3,112, ade1-100, his4-519 and is $\mathrm{GAL}^{+}$) (generously provided by L. Guarente, MIT, Cambridge, MA). The strain used for Dimlp depletion is isogenic except that it has the URA3pGAL10::dim1 construct integrated at the DIM1 locus. Strain BWG1-7A is auxotrophic for uracil; therefore, strain GRF18 (MAT ; leu2-3, 112; his3-11,15, CAN1) (generously provided by R. Serrano, Seville, Spain) was used for pulse-chase labeling of pre-rRNA with $\left[{ }^{3} \mathrm{H}\right]$ uracil. The strain used as the host for pGAL:: rDNA expression is NOY504 (MAT ; rpa12::LEU2; leu2-3, 112; ura3-1; trp1-1; his3-11; CAN1-100) (Nogi et al. 1993; generously provided by M. Nomura, University of California, Irvine, CA).

For Dimlp depletion, cells growing exponentially in galactose minimal medium at $30^{\circ} \mathrm{C}$ were harvested by centrifugation, washed, and resuspended in glucose minimal medium. During growth, cells were diluted with prewarmed medium and constantly maintained in early exponential phase. Identical results were obtained when the experiment was repeated at $37^{\circ} \mathrm{C}$ /data not shown).

For analysis of the $\mathrm{G}_{1779} \mathrm{G}_{1780}$ mutation, strain NOY504 was transformed with plasmid pGAL::rDNA, - rDNA, or pTL2 and grown at $23^{\circ} \mathrm{C}$ in minimal galactose medium before being transferred, at an $\mathrm{OD}_{600}$ of $\sim 0.08$ to $37^{\circ} \mathrm{C}$ for $6 \mathrm{hr}$. For the experiment presented in Figure 11, RNA was extracted following growth at $23^{\circ} \mathrm{C}$; RNA extracted following growth at $18^{\circ} \mathrm{C}$ gave identical results (data not shown).

\section{Construction of a conditional GAL10::diml allele}

A PvuII-XhoI DNA fragment containing the DIM1 ORF, $5^{\prime}$ - and $3^{\prime}$-flanking sequences (Lafontaine et al. 1994) was subcloned into pUC18 to yield plasmid pDL526. In plasmid pDL503, the GAL1-10 promoter region of plasmid pBM272 (Johnston and Davis 1984; kindly provided by M. Johnston, Washington University School of Medicine, St. Louis, MO), isolated by EcoRIBamHI digestion, was subcloned into plasmid pFL44S (Bonneaud et al. 1991) in order to be fused to a URA3 marker. The URA3-pGAL10 cassette was recovered from plasmid pDL503 following a ClaI-EcoRI digestion, filled-in with the Klenow DNA polymerase, and inserted into plasmid pDL526 at the filled ClaI site, which lies 27 nucleotides upstream of the ATG of DIM1. The resulting plasmid, pDL527, was digested with PvuII and the fragment, DIM1 5'-flanking sequence-URA3pGAL10-DIM1 ORF-DIM1 3'-flanking sequence, was purified and used to transform yeast strain BWGl-7A, with selection for uracil prototrophy on galactose minimal medium. One URA ${ }^{+}$ transformant was chosen; integration was checked by PCR and Southern blot analysis (data not shown). All the experiments compared this strain $(G A L:: \operatorname{dim} 1)$ with the otherwise isogenic wild-type $\left\langle D I M 1^{+}\right|$strain.

\section{Construction of the $G_{1770} G_{1771}$ mutant}

Construction of plasmids pGAL::rDNA, - rDNA, pITS1 $\Delta 3^{\prime}$, and pBSrDNAB-XFTag is described in Henry et al. (1994). The $\mathrm{G}_{1779} \mathrm{G}_{1780}$ mutation was produced by two-step PCR. The firstround PCR product, using oligonucleotide 5 -TCGCTAGTACCGATTG-3' and mutagenic oligonucleotide 5'-CCTTCCGCAGGCCCACCTACGG-3', was used as a primer with oligonucleotide $i$ during the second round of amplification. The second-round amplification product was digested with StulDraIII and inserted into plasmid pBSrDNAB-XFTag. An SfiI$X$ hoI fragment was recovered from the resulting plasmid pTL1 and inserted into pGAL::rDNA. The final plasmid was termed pTL2.
RNA extraction, Northern hybridization, primer extension, and pulse-chase labeling

RNA was extracted as described previously (Tollervey and Mattaj 1987). Northern hybridization (Tollervey 1987) and primer extension (Beltrame and Tollervey 1992) were as described previously. For the GAL::dim1 Northern and primer extension experiments, total RNA equivalent to $0.4 \mathrm{OD}_{600}$ of cells $\left(\mathrm{c} 8 \times 10^{6}\right.$ cells) was used for each sample. For the $\mathrm{G}_{1779} \mathrm{G}_{1780}$ Northern blots and primer extension experiments, $9 \mu \mathrm{g}$ of total RNA was used. The primer extension presented in Figure $7 B$, used $\sim 100$ ng of purified $18 \mathrm{~S}$ rRNA per reaction. 18S rRNA was purified from an agarose-TBE gel using DE81 Whatman chromatographic paper. RNA was eluted for $1 \mathrm{hr}$ at $37^{\circ} \mathrm{C}$ in $20 \mathrm{~mm}$ Tris (pH 7.5), $1 \mathrm{~mm}$ EDTA, and $1.5 \mathrm{M} \mathrm{NaCl}$, butanol-extracted, and ethanol-precipitated. The oligonucleotide probes used for Northern hybridization and primer extension are depicted on Figure 1. Oligonucleotide $b$ is GAAAGAAACCGAAATCTC, oligonucleotide $\mathrm{c}$ is CCAGATAACTATCTTAAAAG, oligonucleotide $f$ is TAATGATCCTTCCGCA, and oligonucleotide $g$ is CGGTTTTAATTGTCCTA. Oligonucleotides $a, h, i$, and $k$ are described as B, D, E, and F, respectively, in Morrissey and Tollervey (1993). Oligonucleotides $\mathrm{e}$ and $\mathrm{m}$ hybridize to the tags in the $18 \mathrm{~S}$ and $25 \mathrm{~S}$ rRNAs, respectively, and are described in Beltrame and Tollervey (1992). Oligonucleotide $\mathrm{j}$ hybridizes to the tag in the 5.8S rRNA and is described as fin Henry et al. (1994). Oligonucleotides $\mathrm{d}$ and $\mathrm{l}$ are described as a and $\mathrm{g}$, respectively, in Bergès et al. (1994).

Metabolic labeling of RNA was performed as described previously (Tollervey et al. 1991). Cultures growing exponentially in galactose minimal medium were harvested, washed, and resuspended in prewarmed glucose minimal medium. The cells were subsequently maintained in an early exponential phase by dilution with prewarmed medium. Metabolic labeling with $\left[{ }^{3} \mathrm{H}\right]$ uracil was carried out as described previously (Tollervey et al. 1991) $25 \mathrm{hr}$ after transfer to glucose medium, except that 7 $\mathrm{ml}$ of cells $\left(\mathrm{OD}_{600} \sim 0.3\right)$ were labeled with $233 \mu \mathrm{Ci}$ of label and chase times were $1,2,5,10,20$, and $60 \mathrm{~min}$.

\section{Acknowledgments}

We thank Christine Allmang, Monica Beltrame, Thomas Dandekar, Jean Delcour, Yves Henry, Joe Lewis, Zoi Lygerou, Iain Mattai, Phil Mitchell, John Morrissey, Elisabeth Petfalski, Kishore Sharma, and Jaap Venema for helpful advice and comments on the manuscript. D.L. was the recipient of I.R.S.I.A and European Molecular Biology Organization short-term fellowships.

The publication costs of this article were defrayed in part by payment of page charges. This article must therefore be hereby marked "advertisement" in accordance with 18 USC section 1734 solely to indicate this fact.

\section{References}

Beltrame, M. and D. Tollervey. 1992. Identification and functional analysis of two U3 binding sites on yeast pre-ribosomal RNA. EMBO I. 11: 1531-1542.

Beltrame, M., Y. Henry, and D. Tollervey. 1994. Mutational analysis of an essential binding site for the U3 snoRNA in the $5^{\prime}$ external transcribed spacer of yeast pre-rRNA. Nucleic Acids Res. 22: 5139-5147.

Bergés, T., E. Petfalski, D. Tollervey, and E.C. Hurt. 1994. Synthetic lethality with fibrillarin identifies NOP77p, a nucleolar protein required for pre-rRNA processing and modifica- 
tion. $E M B O$ \%. 13: 3136-3148.

Bonneaud, N., O. Ozier-Kalogeropoulos, G. Li, M. Labouesse, L. Minvielle-Sebastia, and F. Lacroute. 1991. A family of low and high copy replicative, integrative and single-stranded $S$. cerevisiae/E. coli shuttle vectors. Yeast 7: 609-615.

Brand, R.C., J. Klootwijk, T.J.M. van Steenbergen, A.J. de Kok, and R.J. Planta. 1977. Secondary methylation of yeast ribosomal precursor RNA. Eur. J. Biochem. 75: 311-318.

Brimacombe, R., P. Mitchell, M. Osswald, K. Stade, and D. Bochkariov. 1993. Clustering of modified nucleotides at the functional center of bacterial ribosomal RNA. FASEB $J$. 7: 161-167.

Caboche, M. and J.-P. Bachellerie. 1977. RNA methylation and control of eukaryotic RNA biosynthesis. Eur. I. Biochem. 74: 19-29.

Fournier, M.J. and E.S. Maxwell. 1993. The nucleolar snRNAs: Catching up with the spliceosomal snRNAs. Trends Biol. Sci. 18: 131-135.

Girard, J.P., H. Lehtonen, M. Caizergues-Ferrer, F. Amalric, D. Tollervey, and B. Lapeyre. 1992. GARl is an essential small nucleolar RNP protein required for pre-rRNA processing in yeast. $E M B O$ J. 11: 673-682.

Henry, Y., H. Wood, J.P. Morrissey, E. Petfalski, S. Kearsey, and D. Tollervey. 1994. The $5^{\prime}$ end of yeast $5.8 \mathrm{~S}$ rRNA is generated by exonucleases from an upstream cleavage site. $E M B O$ J. 13: 2452-2463.

Hughes, J.M.X. and M.J. Ares. 1991. Depletion of U3 small nucleolar RNA inhibits cleavage in the $5^{\prime}$ external transcribed spacer of yeast pre-ribosomal RNA and impairs formation of 18S ribosomal RNA. EMBO \% 10: 4231-4239.

Jansen, R., D. Tollervey, and E.C. Hurt. 1993. A U3 snoRNP protein with homology to splicing factor PRP4 and G $\beta$ domains is required for ribosomal RNA processing. EMBO $/$. 12: 2549-2558.

Johnston, M. and R.W. Davis. 1984. Sequences that regulate the divergent GAL1-GAL10 promoter in Saccharomyces cerevisiae. Mol. Cell. Biol. 4: 1440-1448.

Klebe, R.J., J.V. Harriss, D. Sharp, and M.G. Douglas. 1983. A general method for poly-ethylene-glycol-induced genetic transformation of bacteria and yeast. Gene 25: 333-341.

Klootwijk, J. and R.J. Planta. 1989. Isolation and characterization of yeast ribosomal RNA precursors and preribosomes. Methods Enzymol. 180: 96-109.

Klootwijk, J., R.C. van den Bos, and R.J. Planta. 1972. Secondary methylation of yeast ribosomal RNA. FEBS lett. 27: 102106.

Lafontaine, D., J. Delcour, A.-L. Glasser, J. Desgrès, and J. Vandenhaute. 1994. The DIM1 gene responsible for the conserved $\mathrm{m}_{2}^{6} \mathrm{Am}_{2}^{6} \mathrm{~A}$ dimethylation in the $3^{\prime}$ terminal loop of $18 \mathrm{~S}$ rRNA is essential in yeast. I. Mol. Biol. 241: 492-497.

Li, H.V., J. Zagorski, and M.J. Fournier. 1990. Depletion of U14 small nuclear RNA (snR128) disrupts production of $18 \mathrm{~S}$ rRNA in Saccharomyces cerevisiae. Mol. Cell. Biol. 10: $1145-1152$.

Lygerou, Z., P. Mitchell, E. Petfalski, B. Séraphin, and D. Tollervey. 1994. The POP1 gene encodes a protein component common to the RNase MRP and RNase P ribonucleoproteins. Genes \& Dev. 8: 1423-1433.

Maden, B.E.H. 1990. The numerous modified nucleotides in eukaryotic ribosomal RNA. Prog. Nucleic Acid Res. Mol. Biol. 39: 241-303.

Morrissey, J.P. and D. Tollervey. 1993. Yeast snR30 is a small nucleolar RNA required for $18 \mathrm{~S}$ rRNA synthesis. Mol. Cell. Biol. 13: 2469-2477.

. 1995. Birth of the snoRNPs-The evolution of RNase MRP and the eukaryotic pre-rRNA processing system.
Trends Biol. Sci. 20: 78-82.

Nogi, Y., R. Yano, J. Dodd, C. Carles, and M. Nomura. 1993. Gene RRN4 in Saccharomyces cerevisiae encodes the A12.2 subunit of RNA polymerase I and is essential only at high temperatures. Mol. Cell. Biol. 13: 114-122.

Raué, H.A. and R.J. Planta. 1991. Ribosome biogenesis in yeast. Prog. Nucleic Acid Res. Mol. Biol. 41: 89-129.

Salim, M. and B. Maden. 1973. Early and late methylations in HeLa cell ribosome maturation. Nature 244: 334-336.

Sirum-Connolly, K. and T.L. Mason. 1993. Functional requirement of a site-specific ribose methylation in ribosomal RNA. Science 262: 1886-1889.

Swann, P.F., A.C. Peacock, and S. Bunting. 1975. Carcinogenesis and cellular injury: The effect of ethionine on ribonucleic acid synthesis in rat liver. Biochem. J. 150: 335-344.

Tollervey, D. 1987. A yeast small nuclear RNA is required for normal processing of pre-ribosomal RNA. EMBO I. 6: 41694175.

Tollervey, D. and I.W. Mattaj. 1987. Fungal small nuclear ribonucleoproteins share properties with plant and vertebrate U-snRNPs. EMBO f. 6: 469-476.

Tollervey, D., H. Lehtonen, M. Carmo-Fonseca, and E.C. Hurt. 1991. The small nucleolar RNP protein NOP1 (fibrillarin) is required for pre-rRNA processing in yeast. $E M B O J$. 10: $573-$ 583.

Tollervey, D., H. Lehtonen, R. Jansen, H. Kern, and E.C. Hurt. 1993. Temperature-sensitive mutations demonstrate roles for yeast fibrillarin in pre-rRNA processing, pre-rRNA methylation, and ribosome assembly. Cell 72: 443-457.

Udem, S.A. and J.R. Warner. 1973. The cytoplasmic maturation of a ribosomal precursor ribonucleic acid in yeast. $/$. Biol. Chem. 248: 1412-1416.

van Buul, C.P.J.J. and P.H. van Knippenberg. 1985. Nucleotide sequence of the $k s g A$ gene of $E$. coli: Comparison of methyltransferases affecting dimethylation of adenosine in ribosomal RNA. Gene 38: 65-72.

van Knippenberg, P.H. 1986. Structural and functional aspects of the $N^{6}, N^{6}$ dimethyladenosines in $16 \mathrm{~S}$ ribosomal RNA. In Structure, function and genetics of ribosomes (ed. B. Hardesty and G. Kramer), pp. 412-424. Springer-Verlag, New York.

van Knippenberg, P.H., J.M.A. van Kimmenade, and H.A. Heus. 1984. Phylogeny of the conserved $3^{\prime}$ terminal structure of the RNA of small ribosomal subunits. Nucleic Acids Res. 12: 2595-2604.

Vaughan, M.H., R. Soeiro, J.R. Warner, and J.E.J. Darnell. 1967. The effects of methionine deprivation on ribosome synthesis in HeLa cells. Proc. Natl. Acad. Sci. 58: 1527-1534.

Warner, J.R. 1989. Synthesis of ribosomes in Saccharomyces cerevisae. Microbiol. Rev. 53: 256-271.

Wolf, S.F. and D. Schlessinger. 1977. Nuclear metabolism of ribosomal RNA in growing, methionine-limited, and ethionine-treated HeLa cells. Biochem. 16: 2783-2791.

Woolford, J.L., Jr. 1991. The structure and biogenesis of yeast ribosomes. Adv. Genet. 29: 63-118.

Woolford, J.L., Jr. and J.R. Warner. 1991. The ribosome and its synthesis. In The molecular and cellular biology of the yeast Saccharomyces: Genome dynamics, protein synthesis and energetics (ed. J.R. Broach, J.R. Pringle, and E.W. Jones), pp. 587-626. Cold Spring Harbor Laboratory Press, Cold Spring Harbor, New York. 


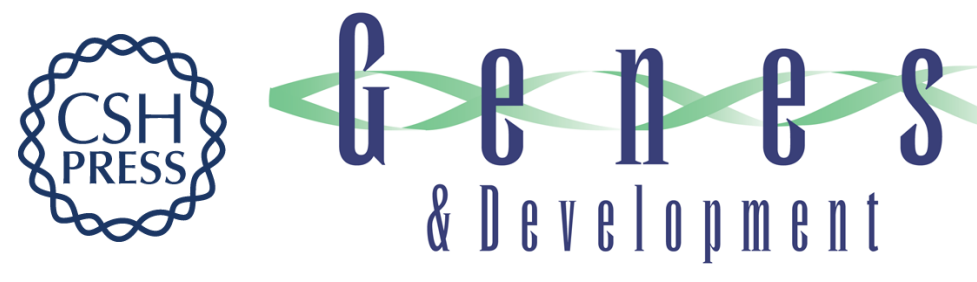

\section{The 18S rRNA dimethylase Dim1p is required for pre-ribosomal RNA processing in yeast.}

D Lafontaine, J Vandenhaute and D Tollervey

Genes Dev. 1995, 9:

Access the most recent version at doi:10.1101/gad.9.20.2470

References This article cites 38 articles, 10 of which can be accessed free at:

http://genesdev.cshlp.org/content/9/20/2470.full.html\#ref-list-1

License

Email Alerting

Service

Receive free email alerts when new articles cite this article - sign up in the box at the top right corner of the article or click here.

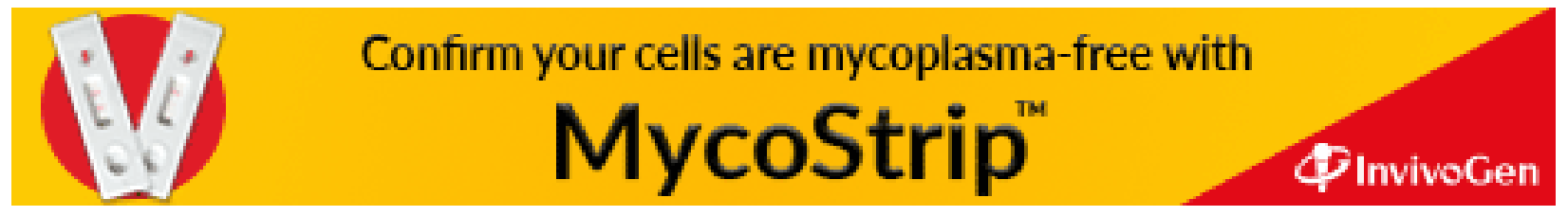

